\title{
REAL-TIME VOLUME RENDERING INTERACTION IN VIRTUAL REALITY
}

\author{
Kosin Kalarat $^{1 *}$, Phanit Koomhin ${ }^{2}$ \\ ${ }^{1}$ School of Informatics, Walailak University, 222 Thaiburi, Thasala District, Nakhon Si Thammarat \\ 80161, Thailand \\ ${ }^{2}$ School of Medicine, Walailak University, 222 Thaiburi, Thasala District, Nakhon Si Thammarat \\ 80161, Thailand
}

(Received: November 2018 / Revised: January 2019 / Accepted: July 2019)

\begin{abstract}
Volume visualization using Direct Volume Rendering (DVR) techniques is used to view information inside 3D volumetric data. Data is classified using a transfer function to emphasize or filter some parts of volumetric information, such as that from Computed Tomography (CT) or Magnetic Resonance Imaging (MRI). In this paper, we introduced an application for real-time volume rendering interaction with 1D transfer functions using Virtual Reality (VR) technology based on the Oculus Rift headset and Oculus Touch controllers. Resulting images were visualized stereoscopically at 60 frames per second using a ray-casting shader, which works based on Graphics Processing Unit (GPU). To evaluate the system, 20 participants interacted with the application to complete three tasks, including a free viewpoint scan, clipping planes renderer, and an editable transfer function in the virtual environment. Then, a survey was carried out using a questionnaire to gather data. Findings showed that the average usability score for the application was 87.54 , which suggested that it was highly usable.
\end{abstract}

Keywords: Direct volume rendering; Ray casting; Virtual environment; Virtual reality

\section{INTRODUCTION}

Volume rendering is an important visualization method in scientific visualization and computer graphics. In medical visualization, Direct Volume Rendering (DVR) has been used to project 3D information from medical data. For example, data from Computed Tomography (CT) or Magnetic Resonance Imaging (MRI) can be reconstructed using reconstruction algorithms, such as back projection or ART or SART algorithms (Kalarat, 2005) to increase diagnostic capabilities and operation planning. To make a medical diagnosis, doctors or radiologists must assess patients' bones, organs, or tumors from a program application display on a personal computer and use a mouse, keyboard, and monitor to control their viewpoint. However, such investigations are limited by the use of 2D screens.

A few years ago, Oculus VR developed and manufactured Oculus Rift, a Virtual Reality (VR) headset with stereoscopic imaging and head tracking. This technology made VR technology popular again due to its effectiveness and low cost. Many large companies have been interested in this technology and have developed and brought to market their own VR headsets, such as High Tech Computer (HTC), Samsung, Sony, Lenovo, and so on, creating a red ocean market. It is a good time for customers and VR developers to obtain these devices, which enable a low cost VR experience. VR technology provides a new way of interacting with virtual information,

*Corresponding author's email: kosin.ka@wu.ac.th, Tel. +66-75-672260

Permalink/DOI: https://doi.org/10.14716/ijtech.v10i7.3259 
especially virtual 3D data.

Applications for VR have been found in a diverse range of fields, such as construction, education, entertainment, the military, medicine and manufacturing (Bahar et al., 2014).

Our team has been interested in the medical applications of DVR in VR. We used the effective head-mounted displays (HMDs) of VR technology currently available to develop an application that increased diagnostic viewpoints when visualizing 3D medical data via volume rendering in an immersive virtual environment. Unlike volume rendering in augmented realities (Kutter et al., 2008) which are in the real environment, in a virtual environment, users can interact with 3D medical data in real-time by rotating it or changing viewpoints. Our VR application was developed to run on an Oculus Rift headset and Oculus Touch controller, which was used to interact with clinical data. Unity3D, real-time development platform, was used to manage the interaction, render pipeline, and program the relevant shader to parallelize the time-consuming ray-casting algorithm. For this research, we developed a VR application to visualize medical data using a DVR technique on VR technology focusing on system interaction. Three main features, which included a free viewpoint scan, clipping plane renderer, and an editable transfer function, were used to evaluate the performance and usability of the VR application.

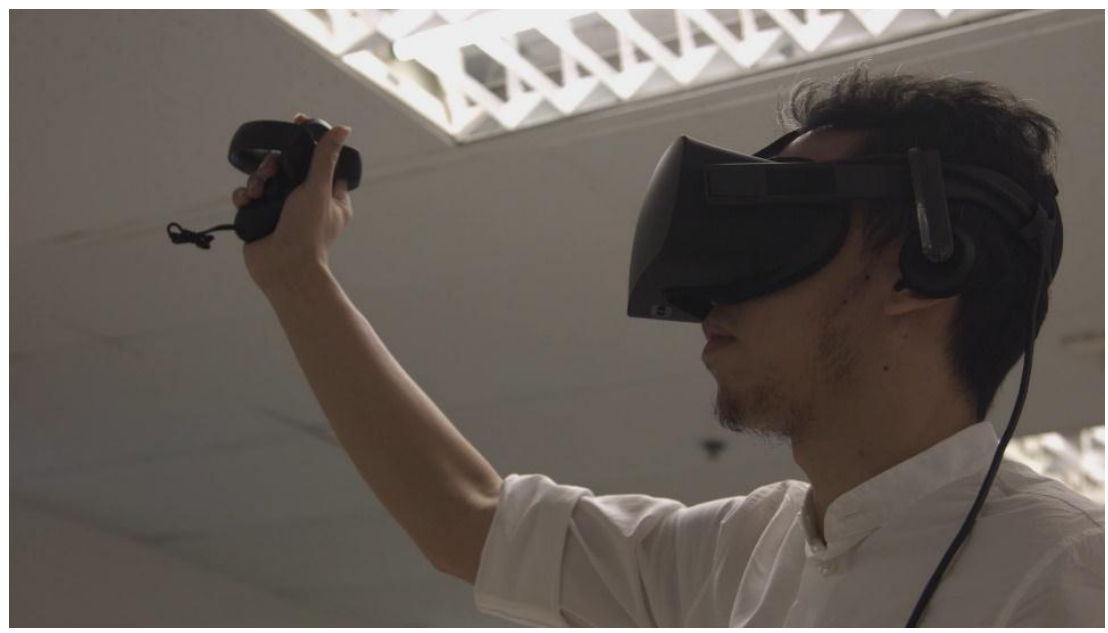

Figure 1 Virtual reality using an Oculus Rift headset and Oculus Touch controller

\section{BACKGROUND}

\subsection{Volume Rendering}

Volume rendering (Drebin et al., 1988) is an important data visualization method, which is predominantly used in the fields of scientific and medical visualization. Typically, volumetric data sets in volume rendering comprise many 2D slice images acquired by a CT, MRI, or MicroCT scanner. 2D slice images slices are arranged as a volumetric grid by converting their pixels to voxels to acquire a single value using the value of surrounding pixels.

After obtaining 3D volumetric data, the rendering system must define a camera relative to the 3D data set to determine rendering properties, for example, the opacity and color of each voxel. Then, a ray-casting method is used to simulate the projection of a camera ray through the volumetric data and calculate for each ray through each image pixel. This process usually includes a transfer function, which applies Red, Green, Blue, Alpha (RGBA) to define the RGBA value of every possible voxel value. The transfer function separates bone, tissues, and soft tissues depending on data density accumulation and projects each ray to the $2 \mathrm{D}$ image plane for rendering. Rendering data is conducted by controlling the camera angle and position for the considered viewpoint. 
For this research, we used DVR to display volume data, as it shows cross-sectional images and can render any point of view for radiology diagnosis in a VR environment.

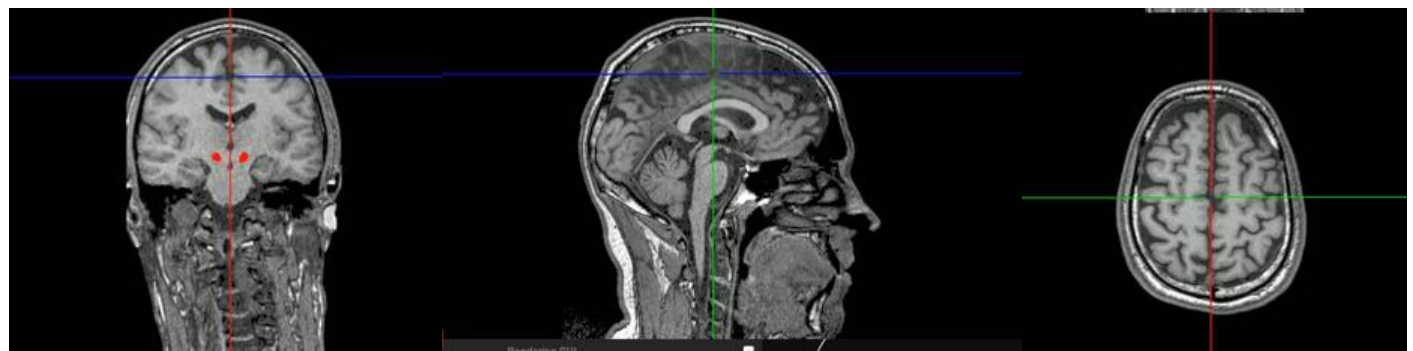

Figure 2 Volume renderings of sagittal, coronal, and transverse anatomical planes of a human head

\subsection{Virtual Reality}

VR (Sherman \& Craig, 2003) is an artificial environment that interacts with a computer to simulate participants' senses, such as their sense of position, and replaces or augments feedback to one or more of them. It provides a feeling of immersion or presence in a simulation in some situations (Kalarat, 2014). Generally, VR is a fully synthetic environment comprising artificial imagery that can impinge on users' senses, whether that imagery is visual, auditory, haptic, and so on. VR systems can address participants' location and orientation to create an appropriate point of view in the display.

There are many kinds of VR, which can be divided by the level of immersion they offer: fullyimmersive VR, semi-immersive VR, and non-immersive VR (Fons et al., 2018). Normally, VR technology is fully immersive and uses headsets or multi-projected environments. In some cases, it is combined with physical environments or props to generate realistic images, sounds, and other sensations that simulate a user's physical presence in a virtual or imaginary environment. This immersive environment can be similar to the real world, or it can be fantastical. Moreover, it can simulate specific situations used to practice or train users to encounter a real-life situation correctly, such as virtual endoscopy (Scharsach et al., 2006).

For this research, we used fully-immersive VR and participants used an HMD and hand controllers to interact with DVR data by changing their viewpoint and selecting cross-sectional images.

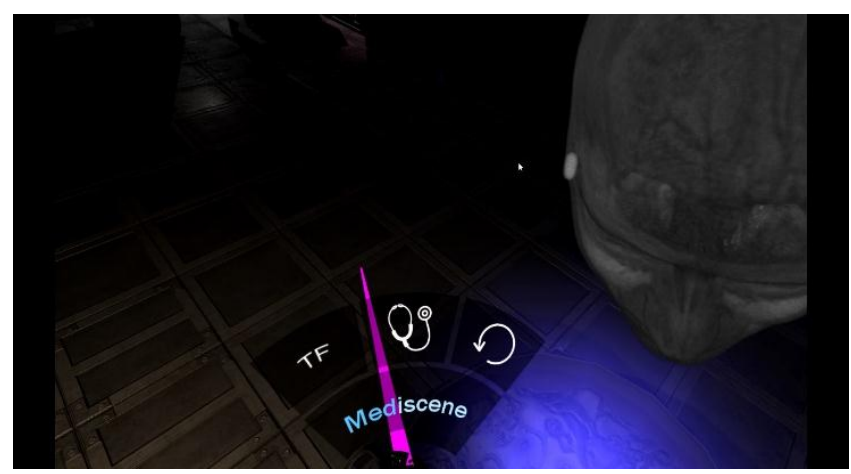

Figure 3 Virtual menu provided in the virtual environment for volume rendering interaction

\section{METHODS}

\subsection{Direct Volume Rendering}

DVR is used to create a 3D representation of volume data directly. Data represents a semitransparent light-emitting medium. Therefore, gaseous phenomena can also be simulated. DVR approaches are based on the laws of physics (emission, absorption, and scattering). Volume data 
is used as a whole, meaning that we can look inside the volumetric data and see all interior structures. In DVR, either backward or forward methods can be used. Backward methods use image space/image order algorithms and are performed pixel-by-pixel. An example is ray casting, which will be discussed in detail below. Forward methods use object space/object order algorithms, which are performed voxel-by-voxel, and cells are projected onto the image. Examples of this technique include slicing, shear-warp, and splatting.

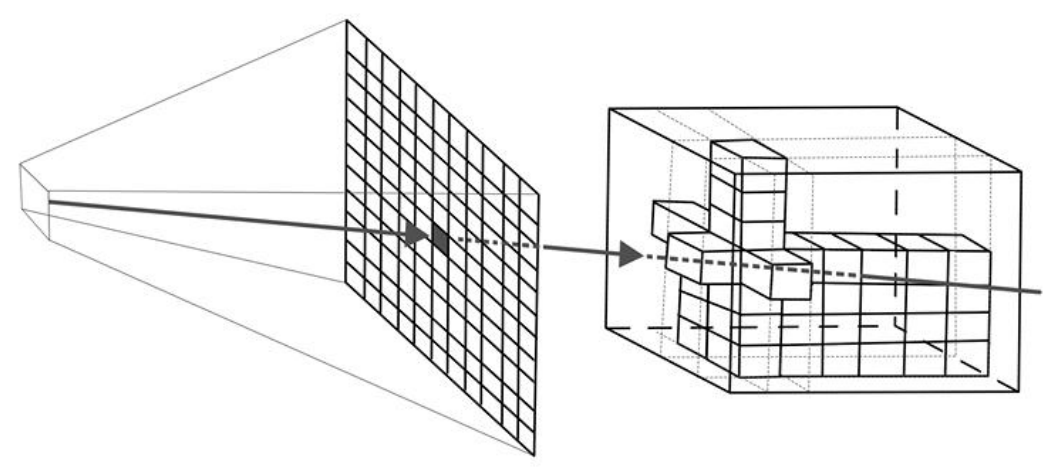

Figure 4 Ray casting using backward methods

Ray casting is similar to ray tracing in surface-based computer graphics. In volume rendering, we only deal with primary rays, which can spawn other rays, such as secondary rays that are only used for shadows, reflection, or refraction. Ray casting is a natural image order technique. As we have no surfaces in DVR, we must step carefully through the volume. A ray is cast into the volume, sampling the volume at certain intervals. Sampling intervals are usually equidistant, but in some types of sampling, such as importance sampling, they are not. At each sampling location, a sample is interpolated/reconstructed from the voxel grid as shown in Figure 4. Popular filters include the nearest neighbor (box), trilinear, or more sophisticated Gaussian or Cubic Spline.

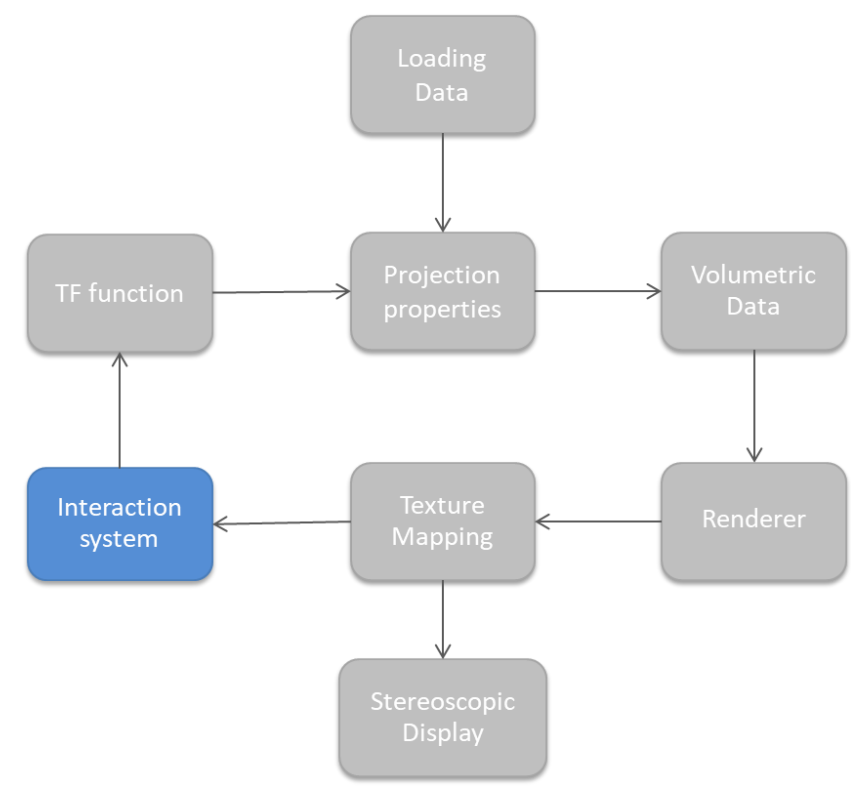

Figure 5 Virtual reality interaction flowchart

Figure 5 shows a flowchart of volume rendering in a real-time interaction. It starts when the MRI or CT data set is loaded to the application, and projection parameters are defined to set the rendering plan. After projection parameters have been set, the image will appear when the rendering method is finished. The ray casting computes the volume-rendering integral by 
sampling along each ray to fragment the shader processing on the GPU (Heng et al., 2006). The resulting image will be mapped as texture and display in the stereoscopic headset. The application will wait for new input, such as changes to the point of view or transfer function value, and will send new projection parameters accordingly.

\subsection{VR Interaction}

For this research, the VR application was displayed in an Oculus Rift headset for head tracking and a stereoscopic viewpoint. Oculus Rift renders two images consistently via stereoscopic imaging and tracks the movement of a user's head in six Degrees of Freedom (DoFs). This means that stereoscopic imaging displays simultaneously, as users change viewpoint by moving their head.

The Oculus Touch system comprises two hand controllers that are used to change modes in the application by pointing a virtual laser to a virtual user interface using the right-hand controller. In addition, when a user selects the free viewpoint scan, the right-hand controller will change shape to a rectangle to prepare itself to scan the CT or MRI data in six DoF. Three interaction features were used to evaluate the system's usability, which included a free viewpoint scan, clipping planes renderer, and an editable transfer function.

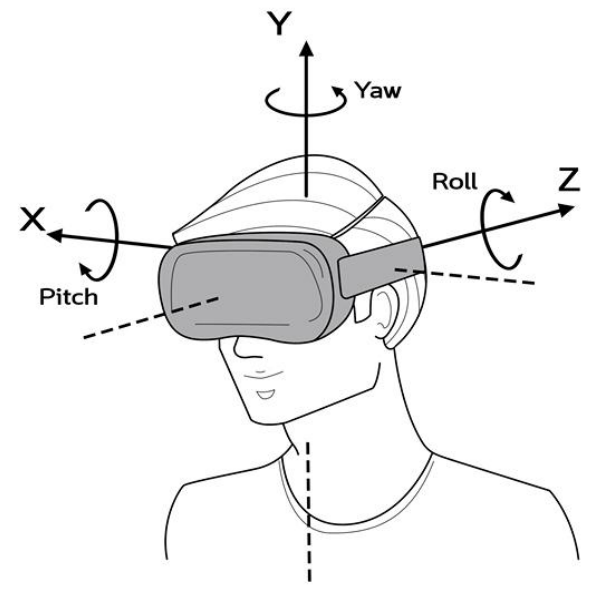

Figure 6 Head tracking in six degrees of freedom corresponds to real-time head movements

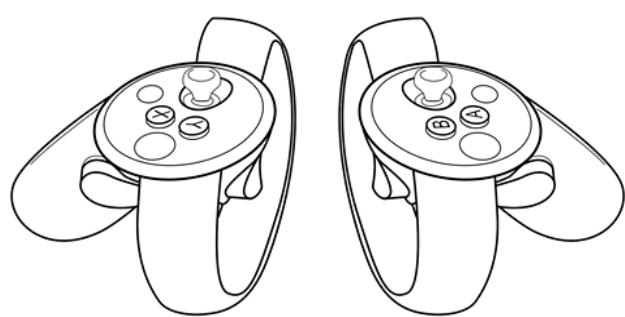

Figure 7 Oculus Touch controllers for virtual environment interaction

Free Viewpoint Scan: This mode allows participants to use Oculus' headset handles to track each movement of the headset and display a stereoscopic image in real-time with a new free axis control using the Oculus Touch controllers. The right hand controller generates virtual controls with rectangular shapes to simulate a scanner. Participants can use the rectangle to touch the medical data, which will be clipped in whatever axis they have touched as shown in Figure 8a.

Clipping planes renderer: This feature allows users to view different anatomical planes. In the virtual user interface, participants can use a virtual laser via the Oculus Touch controller to select the anatomical plane they want to view. Users can move along the provided axes, which allow 
them to view sagittal (left-to-right), transverse (top-to-bottom), and coronal (front-to-back) planes as shown in Figure 8b.

Transfer Function Editor: This feature was designed with 1D transfer function, which allows users to change the transfer function from the virtual user interface to the medical data rendered. Users can change the data color and opacity of their desired viewpoint and view the volume visualization effect in VR in real-time. This helps them find the best classification for separation materials as shown in Figure 8c.

\subsection{Implementation}

The VR application was developed using Unity3D, which is a game engine for interaction and visualization using $256 \times 256 \times 213$ MRI volumetric data. We used Unity 2018.1 to implement a single-pass ray-casting shader in CG language ( $\mathrm{C}$ programming language for graphics), which is a high-level shading language developed by Nvidia. The application for this research was run on an Intel Core i7-7700HQ CPU and NVIDIA GeForce GTX 1060 with 16 GB of RAM.

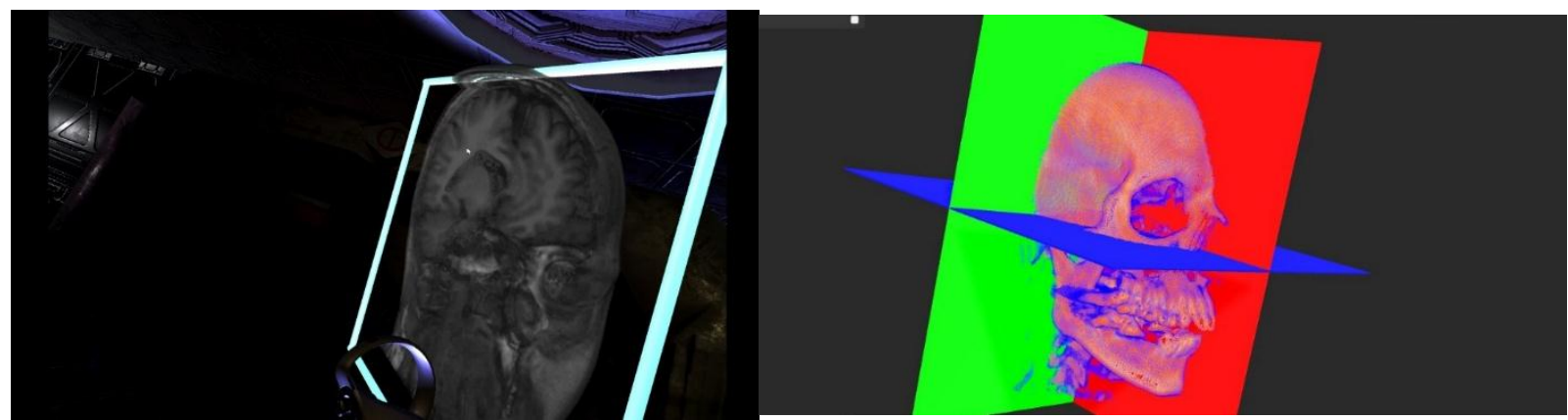

(a)

(b)

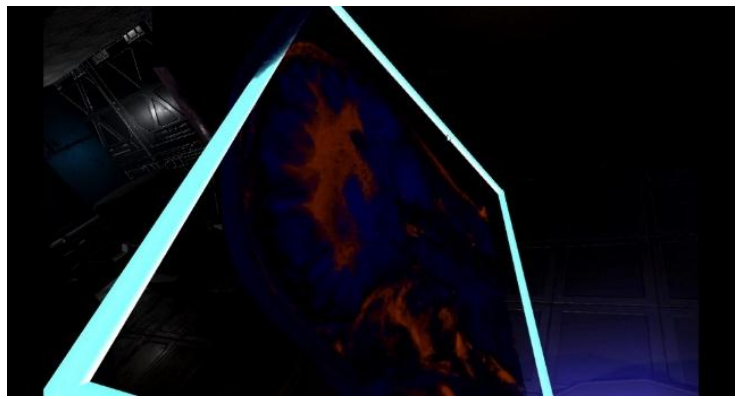

(c)

Figure 8 The resulting images of three interaction features: (a) free viewpoint scan; (b) clipping planes renderer; and (c) transfer function editor

\section{RESULTS AND DISCUSSION}

This research evaluated the performance and usability of a VR application. To evaluate the VR performance of the application, we counted the frame rate rendered by the Unity3D platform. The system provided stereoscopic images at 60 frames per second, which is a standard frame rate in VR. As such, users experienced smooth and continuous images during viewpoint changes that occurred when they moved the HMD with their head. When users changed viewpoint, the application rendered the new image simultaneously in real-time, which meant that the virtual camera re-projected and ran the backward method to calculate the value of the voxels in ray casting to make a new projection image. The application used a game engine to provide capabilities for GPU (Kruger \& Westermann, 2003) ray casting, which can calculate each ray in parallel and map the final pixel value to the projection plan in the shader.

To evaluate interaction, 20 participants were asked to rate the usability of the application on a 7-point Likert scale from 1 (very difficult) to 7 (very easy). We used three tasks in the evaluation, 
which were a free viewpoint scan, clipping planes renderer, and an editable transfer function. Usability results have been shown in Table 1 . The free viewpoint scan was a very easy task, because users can only use the controller to scan inside the volumetric data. In contrast, the other tasks are quite difficult to complete and understand, as all participants were inexperienced in the field of radiology.

Table 1 Evaluation Results

\begin{tabular}{llcc}
\hline \multicolumn{1}{c}{ Task } & Mean value & Std. dev \\
\hline 1. & Free viewpoint scan & 6.85 & 4.20 \\
2. Clipping planes & 6.35 & 3.10 \\
3. Editable transfer function & 6.20 & 2.85 \\
\hline
\end{tabular}

Table 1 shows mean usability scores for different tasks. Usability scores from a total of 20 participants corresponded to a 1 to 7 Likert scale value from 1 (very difficult) to 7 (very easy). This research used the System Usability Scale (SUS; Brooke, 1996), which measures usability. The SUS is an eight-item questionnaire, including four positive and negative response options. In this study, the 20 users who tested the VR application had no experience in using VR or in volume rendering data. However, minimum, mean, and maximum SUS scores were 72.0, 87.54, and 90.75 , respectively. The mean SUS score of 87.54 suggested that our application had very good usability.

\section{CONCLUSION}

In this paper, we have presented the new way interaction with volume rendering in immersive Virtual Environment using Oculus Rift and its controller applying for medical volumetric data from MRI and CT-Scan. The interaction includes 3 features which are free viewpoint scan, clipping planes and editable transfer function.

The application evaluation result for the performance and usability shows that the VR application is able to provide the volumetric data smoothly and intuitively with real-time interaction. Because of sufficient frame rate, users have no motion sickness from the stereoscopic rendering of volume data and they have the freedom to see every part of data with the free viewpoint in real-time. Moreover, the application allows the user to use the Oculus's controller to interact with volumetric data easily, even for inexperienced users. However, this research has been in the early stage of interactive evaluation as the usability tests were tested by the participants, who are not involved in radiological technology. Therefore, this application will be evaluated by the professional radiologist or person who concerned about this field.

In the future, we would evaluate the usability of these features for the VR application with the specific group of users such as radiological technologists or medical students who are studying in the subject of radiology for the aspect of the interactive diagnostic radiology in Virtual Reality.

\section{ACKNOWLEDGEMENT}

This research is supported by VR Inventors project from DEPA (Digital Economy Promotion Agency), Brain-science and Artificial intelligence research unit, and School of Informatics, Walailak University.

\section{REFERENCES}

Bahar, Y.N., Landrieu, J., Pere, C., Nicolle, C., 2014. Simulation and Visualization of Thermal Metaphor in a Virtual Environment for Thermal Building Assessment. International Journal of Technology, Volume 5(1), pp. 3-13 
Brooke, J., 1996. SUS-A Quick and Dirty Usability Scale. Usability Evaluation in Industry, Volume 189(194), pp. 4-7

Drebin, A.R., Carpenter L., Hanrahan P., 1988. Volume Rendering. ACM Siggraph Computer Graphics, Volume 22(4), pp. 65-74

Fons, J., Monclús Lahoya, E., Vázquez Alcocer, P., Navazo Álvaro, I., 2018. Rendering and Interacting with Volume Models in Immersive Environments. In: CEIG 18: XXVIII Spanish Computer Graphics Conference: Madrid, Spain, June 27-29, 2018, European Association for Computer Graphics (Eurographics), pp. 47-50

Heng, Y., Gu, L., 2006. GPU-based Volume Rendering for Medical Image Visualization. In: 2005 IEEE Engineering in Medicine and Biology $27^{\text {th }}$ Annual Conference, pp. 5145-5148

Kalarat, K., 2014. Relief Mapping on Facade of Sino Portuguese Architecture in Virtual Reality. In: 2014 Fourth International Conference on Digital Information and Communication Technology and its Applications (DICTAP), pp. 333-336

Kalarat, K., Narkbuakaew, W., Pintavirooj, C., Sangworasil, M., 2005. Rapid Simultaneous Algebraic Reconstruction Technique (SART) for Cone-beam Geometry on Clustering System. In: TENCON 2005-2005 IEEE Region 10 Conference, pp. 1-4

Kruger, J., Westermann, R., 2003. Acceleration Techniques for GPU-based Volume Rendering. In: Proceedings of the $14^{\text {th }}$ IEEE Visualization 2003 (VIS'03), pp. 287-292

Kutter, O., Aichert, A., Bichlmeier, C., Traub, J., Heining, S.M., Ockert, B., Euler E., Navab, N., 2008. Real-time Volume Rendering for High Quality Visualization in Augmented Reality. In: International Workshop on Augmented environments for Medical Imaging including Augmented Reality in Computer-aided Surgery (AMI-ARCS 2008), New York, USA, pp. 104-113

Scharsach, H., Hadwiger, M., Neubauer, A., Wolfsberger, S., Bühler, K., 2006. Perspective Isosurface and Direct Volume Rendering for Virtual Endoscopy Applications. In: Eurovis, Volume 6, pp. 315-322

Sherman, W.R., Craig, A.B., 2003. Understanding Virtual Reality: Interface, Application, and Design. Morgan Kaufmann. USA, pp. 4-16 